\title{
An Ineffective Differential Diagnosis of Infective Endocarditis and Rheumatic Heart Disease after Streptococcal Skin and Soft Tissue Infection
}

\author{
Tetsuya Suzuki ${ }^{1,2}$, Momoko Mawatari ${ }^{2,3}$, Toshihiko Iizuka ${ }^{4}$, Tatsuya Amano ${ }^{5}$, \\ Satoshi Kutsuna ${ }^{2}$, Yoshihiro Fujiya ${ }^{2}$, Nozomi Takeshita ${ }^{2}$, \\ Kayoko Hayakawa ${ }^{2}$ and Norio Ohmagari ${ }^{2}$
}

\begin{abstract}
:
We herein report the case of a 68-year-old woman with a skin and soft tissue infection at her extremities. The blood culture results were positive for Streptococcus pyogenes, and we started treatment using ampicillin and clindamycin, although subsequent auscultation revealed a new-onset heart murmur. We therefore suspected rheumatic heart disease and infective endocarditis. The case met both the Jones criteria and the modified Duke criteria. Transesophageal echocardiography revealed vegetation on the aortic valve, although the pathological findings were also compatible with both rheumatic heart disease and infective endocarditis. The present findings suggest that these two diseases can coexist in some cases.
\end{abstract}

Key words: Streptococcus pyogenes, skin and soft tissue infection, infective endocarditis, modified Duke's criteria, acute rheumatic fever, Jones criteria

(Intern Med 56: 2361-2365, 2017)

(DOI: 10.2169/internalmedicine.8411-16)

\section{Introduction}

Streptococcus species are well-known bacteria that cause skin and soft tissue infections, as well as bacterial tonsillitis. Clinicians should be concerned if the patient develops an acute rheumatic fever after a streptococcal infection, as the disease has an unpredictable course. In these cases, the diagnosis is made based on the Jones criteria, which include five major manifestations (migratory polyarthritis, carditis and valvulitis, chorea, subcutaneous nodules, and erythema marginatum) and four minor manifestations (arthralgia, a fever, elevated acute-phase reactants, and a prolonged PR interval during electrocardiography) $(1,2)$. In cases of a confirmed previous streptococcal infection, two major manifestations or one major manifestation plus two minor manifestations are sufficient to diagnose an acute rheumatic fever $(1,2)$. Pha- ryngitis is typically the cause of an acute rheumatic fever, which is most frequently observed children, although adults can develop acute rheumatic disease, and these cases are infrequently caused by a streptococcal skin infection $(3,4)$.

Infective endocarditis is a serious complication after Gram-positive coccal bacteremia, and the key clinical signs include a sustained fever, a new-onset heart murmur, and/or peripheral embolic signs. This condition is diagnosed based on the modified Duke criteria, which include two major criteria (positive blood culture results for typical microorganisms and/or evidence of endocardial involvement, such as vegetation, abscess, or new valvular regurgitation) and five minor criteria (predisposing factors, a fever, vascular phenomena, immunological phenomena, and microbiological findings that do not meet the major criteria or serological evidence of active infection with an organism that is consistent with infective endocarditis) (5). A definitive diagnosis

\footnotetext{
${ }^{1}$ Department of Internal Medicine, Nerima Hikarigaoka Hospital, Japan, ${ }^{2}$ Disease Control and Prevention Center, National Center for Global Health and Medicine, Japan, ${ }^{3}$ Infection Control and Prevention Center, Gunma University Hospital, Japan, ${ }^{4}$ Department of Diagnostic Pathology, National Center for Global Health and Medicine, Japan and ${ }^{5}$ Department of Cardiology, New Tokyo Hospital, Japan Received: October 16, 2016; Accepted: January 24, 2017; Advance Publication by J-STAGE: August 10, 2017 Correspondence to Dr. Momoko Mawatari, mawatarimo@gunma-u.ac.jp
} 
of infective endocarditis can be made based on two major criteria, one major criterion plus three minor criteria, or five minor criteria; a possible diagnosis can be made based on one major criterion and one minor criterion, or three minor criteria (5). Streptococcus species are an uncommon cause of infective endocarditis (6), although a new-onset heart murmur after streptococcal bacteremia may indicate that the patient has infective endocarditis. Furthermore, it is possible to develop both an acute rheumatic fever and infective endocarditis after streptococcal bacteremia.

We herein report a case in which it was difficult to differentiate between infective endocarditis and an acute rheumatic fever after a streptococcal skin and soft tissue infection.

\section{Case Report}

A 68-year-old Japanese woman was admitted with severe pain in her upper and lower extremities. At 14 days before the admission, she had fallen and sustained injuries to her left palm and right elbow. At 10 days before the admission, she experienced abdominal pain, nausea, and diarrhea. On the day of admission, a neighbor called an emergency medical service after finding the patient lying on the floor of her house. The patient had a history of endometrial cancer and hepatitis $\mathrm{C}$, and her vital signs at admission were a body temperature of $38.8^{\circ} \mathrm{C}$, a heart rate of $118 \mathrm{bpm}$, a blood pressure of $128 / 69 \mathrm{mmHg}$, a respiratory rate of $20 / \mathrm{min}$, and an arterial blood oxygen saturation $\left(\mathrm{SpO}_{2}\right)$ of $95 \%$ in room air. A physical examination also revealed a systolic heart murmur on the third intercostal left sternal border, and her right upper arm and left knee were red, swollen, and tender. However, we did not detect any splinter hemorrhaging, Osler's nodes, or Janeway lesions. Laboratory testing revealed leukocytosis $(26,830 / \mu \mathrm{L})$ and elevated levels of C-reactive protein $(36.46 \mathrm{mg} / \mathrm{dL})$, aspartate transaminase (40 IU/L), alanine transaminase $(29 \mathrm{IU} / \mathrm{L})$, blood urea nitrogen $(74.2$ $\mathrm{mg} / \mathrm{dL})$, and creatinine $(1.94 \mathrm{mg} / \mathrm{dL})$. Two sets of blood cultures were taken at the emergency department. Electrocardiography revealed a normal sinus rhythm without ST-T segment change. There was no apparent vegetation on the transthoracic echocardiogram obtained at admission, and chest and abdominal computed tomography revealed normal findings, with the exception of bilateral renal stones.

On day 3, arthrocentesis was performed on the patient's left knee. Gram staining revealed streptococcus-like Grampositive cocci, although a culture of the synovial fluid revealed negative findings. The two sets of blood cultures from the admission revealed growth of Streptococcus pyogenes. The patient's tender and erythematous extremities, combined with the arthrocentesis results, strongly suggested that she had developed infective arthritis secondary to bacteremia from a streptococcal skin and soft tissue infection. Therefore, we started treatment using ampicillin ( 2 g every 6 hours) and clindamycin (600 mg every 8 hours). On days 5 and 13, after starting the antibiotic treatment, we collected two pairs and one pair of blood cultures respectively, and observed negative results for bacterial growth. Lavage and debridement of the left knee was performed on day 8. The streptococcus-like Gram-positive cocci had vanished completely (based on Gram staining), and culture of the left knee synovial fluid also provided negative results. However, the patient reported persistent focal pain and warmth at her left knee, and we detected a mild fever $\left(37-38^{\circ} \mathrm{C}\right)$.

On day 4, computed tomography had revealed new-onset pleural effusion and ascites, which were not present at the initial evaluation. Based on these findings, we also considered serositis as a clinical manifestation of an autoimmune reaction, such as a rheumatic fever. Aspirin was added as a treatment for the arthritis and fever, which partially relieved the patient's symptoms, and the ascites disappeared. However, the pleural effusion gradually expanded, and auscultation on day 18 revealed a newly-onset diastolic murmur on the second intercostal right sternal border. A transthoracic echocardiogram also revealed aortal regurgitation without vegetation. The ejection fraction was $68.7 \%$. We therefore suggested performing transesophageal echocardiography, although the patient would not consent to this invasive examination. Her heart failure gradually worsened, despite the use of diuretics. Her urine output suddenly decreased to 130 $\mathrm{mL} /$ day on day 50 .

Under suspicion of drug-induced acute kidney injury, antibiotics (amoxicillin $500 \mathrm{mg} 4$ times daily) and aspirin (500 mg 3 times daily) were immediately discontinued on day 51. Her urine output spontaneously recovered, and she did not exhibit elevated creatinine levels. She ultimately consented to transesophageal echocardiography on day 55. This examination revealed vegetation and prolapse of the aortic valve, and we performed aortic valve replacement on day 56. The pathological findings revealed massive neutrophil infiltration with fibrin deposition, which indicated strong inflammation, such as infective endocarditis (Fig. 1). We were unable to detect Aschoff bodies, which are specific to a rheumatic fever. Gram staining and culture of the valve specimen did not reveal any bacteria. The patient has experienced a good recovery since the surgery and subsequent antibiotic treatment. The clinical course is shown in Fig. 2.

\section{Discussion}

Streptococcus pyogenes is an uncommon cause of infective endocarditis, accounting for only approximately $5 \%$ of cases (6). In this context, clinicians diagnose streptococcal infective endocarditis using the modified Duke criteria and results from previous blood cultures positive for Streptococcus pyogenes $(7,8)$. Acute rheumatic fever and rheumatic heart disease are also major complications of streptococcal infections. The incidence of an acute rheumatic fever is highest among 5- to 14-year-old children, although it can also occur among patients over 45 years of age (9). Reports from before 1961 estimated that $1.7 \%$ of patients who did not receive antibiotics for streptococcal pharyngitis subse- 

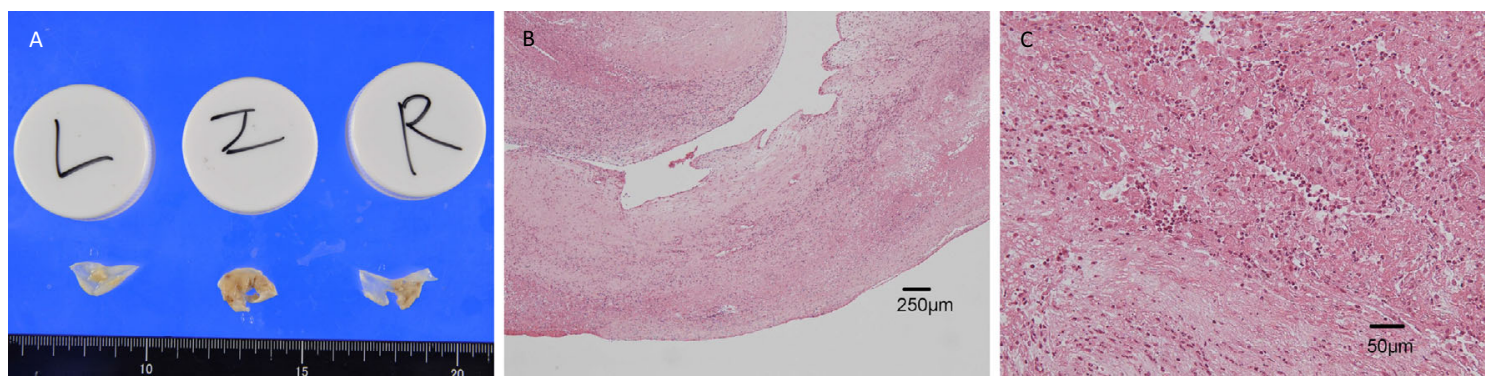

Figure 1. A: Macroscopic view of the aortic valve specimen. Right coronary cusp, noncoronary cusp, and left coronary cusp (from the left). B: A magnified view of the noncoronary cusp (4×). C: A magnified view of the noncoronary cusp $(20 \times)$. The pathological findings revealed massive neutrophil infiltration with fibrin deposition.

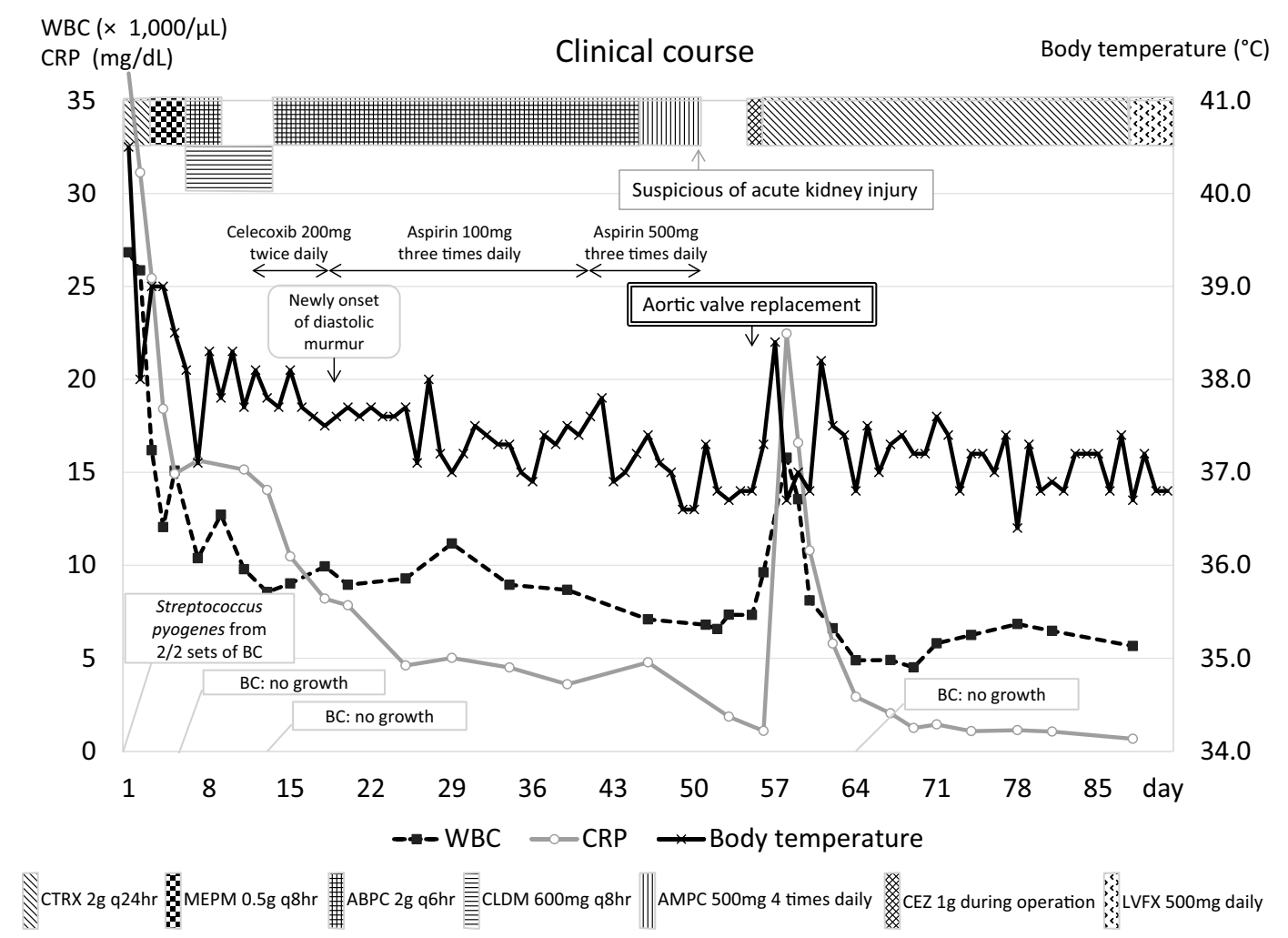

Figure 2. Clinical course of the patient. WBC: white blood cell, CRP: C-reactive protein, BC: blood culture, CTRX: ceftriaxone, MEPM: meropenem, ABPC: ampicillin, CLDM: clindamycin, AMPC: amoxicillin, CEZ: cefazolin, LVFX: levofloxacin, q: every, hr: hour

quently developed an acute rheumatic fever, although its incidence has declined in recent decades (10). While previous reports have only described streptococcal pharyngitis as being responsible for the onset of these diseases, recent reports have also described streptococcal skin infections that resulted in an acute rheumatic fever and rheumatic heart disease $(3,4)$.

In the present case, two sets of blood cultures obtained at admission were positive for Streptococcus pyogenes, and a skin and soft tissue infection was considered the most likely entry site. Given the new-onset diastolic heart murmur that we detected after treating the streptococcal skin and soft tissue infection, the most likely diagnoses appeared to be a rheumatic fever or infective endocarditis. The patient met one major criterion and two minor criteria from the Jones criteria for a rheumatic fever $(1,2)$, suggesting the possibility of an acute rheumatic fever, despite the patient not being from an endemic population. However, the patient also met one major criterion and one minor criterion from the modified Duke criteria (5), suggesting the possibility of infective endocarditis. The patient therefore met the criteria for both a rheumatic fever and infective endocarditis. Our pathological findings from the heart valve specimen were compatible with infective endocarditis. The finding of acute phase inflammatory changes despite prolonged antibiotic therapy might be explained by the ineffectiveness of the conservative treatment for invasive infective endocarditis. The clinical course of the arthritis and fever relief after aspirin treatment 
Table. A Summary of Cases of Group A Streptococcal Endocarditis and Whether They Fulfilled the Jones Criteria.

\begin{tabular}{|c|c|c|c|c|c|c|c|c|c|}
\hline \multirow{2}{*}{$\begin{array}{l}\text { Case } \\
\text { no. }\end{array}$} & \multirow[b]{2}{*}{ Reference } & \multirow{2}{*}{$\begin{array}{l}\text { Patient's } \\
\text { age, sex }\end{array}$} & \multirow{2}{*}{$\begin{array}{l}\text { Identification } \\
\text { of Group A } \\
\text { streptococcus }\end{array}$} & \multirow{2}{*}{$\begin{array}{l}\text { Major } \\
\text { criteria }\end{array}$} & \multicolumn{4}{|c|}{ Minor criteria } & \multirow{2}{*}{$\begin{array}{l}\text { Jones Criteria, } \\
1992 \text { update }\end{array}$} \\
\hline & & & & & Arthralgia & Fever & $\begin{array}{l}\text { Elevated acute } \\
\text { phase reactant }\end{array}$ & $\begin{array}{l}\text { Prolonged PR } \\
\text { interval }\end{array}$ & \\
\hline 2 & [12] & $4 \mathrm{y}, \mathrm{F}$ & Blood culture & $\begin{array}{l}\text { Endocarditis, } \\
\text { polyarthritis }\end{array}$ & + & + & Not shown & Not shown & Sufficient \\
\hline 3 & [13] & $52 \mathrm{y}, \mathrm{M}$ & Blood culture & Endocarditis & + & + & + & Not shown & Sufficient \\
\hline 4 & {$[13]$} & $63 \mathrm{y}, \mathrm{F}$ & Blood culture & Endocarditis & Not shown & + & + & Not shown & Sufficient \\
\hline 5 & [13] & $65 \mathrm{y}, \mathrm{F}$ & Blood culture & Endocarditis & Not shown & + & + & Not shown & Sufficient \\
\hline 6 & [13] & $69 \mathrm{y}, \mathrm{F}$ & Blood culture & Endocarditis & Not shown & - & Not shown & Not shown & Not sufficient \\
\hline 7 & [13] & $55 \mathrm{y}, \mathrm{M}$ & Blood culture & Endocarditis & Not shown & + & Not shown & Not shown & Not sufficient \\
\hline 8 & [14] & $3 \mathrm{y}, \mathrm{F}$ & Blood culture & Endocarditis & Not shown & + & Not shown & Not shown & Not sufficient \\
\hline 9 & [15] & $5 \mathrm{mo}, \mathrm{M}$ & Blood culture & Endocarditis & Not shown & + & Not shown & Not shown & Not sufficient \\
\hline 10 & [16] & $4 \mathrm{y}, \mathrm{M}$ & Blood culture & Endocarditis & Not shown & + & Not shown & Not shown & Not sufficient \\
\hline 12 & [17] & $16 \mathrm{y}, \mathrm{F}$ & Blood culture & Endocarditis & Not shown & + & + & Not shown & Sufficient \\
\hline 13 & [18] & $68 \mathrm{y}, \mathrm{M}$ & Blood culture & Endocarditis & Not shown & + & + & Not shown & Sufficient \\
\hline 14 & [7] & $64 \mathrm{y}, \mathrm{M}$ & Blood culture & Endocarditis & Not shown & + & + & Not shown & Sufficient \\
\hline 15 & [19] & $55 \mathrm{y}, \mathrm{M}$ & $\begin{array}{l}\text { Blood culture, } \\
\text { joint fluid, } \\
\text { synovial fluid }\end{array}$ & $\begin{array}{l}\text { Endocarditis, } \\
\text { polyarthritis }\end{array}$ & + & + & + & Not shown & Sufficient \\
\hline 16 & [20] & $69 \mathrm{y}, \mathrm{F}$ & Blood culture & Endocarditis & Not shown & + & Not shown & Not shown & Not sufficient \\
\hline 17 & {$[8]$} & $71 \mathrm{y}, \mathrm{M}$ & Blood culture & Endocarditis & Not shown & + & + & Not shown & Sufficient \\
\hline 18 & [21] & $6 \mathrm{y}, \mathrm{F}$ & Blood culture & Endocarditis & Not shown & + & Not shown & Not shown & Not sufficient \\
\hline 19 & [21] & $8 \mathrm{mo}, \mathrm{F}$ & Blood culture & Endocarditis & Not shown & + & Not shown & Not shown & Not sufficient \\
\hline 20 & {$[22]$} & $42 \mathrm{y}, \mathrm{M}$ & Blood culture & Endocarditis & + & + & + & Not shown & Sufficient \\
\hline
\end{tabular}

y: years, mo: months, M: male, F: female

was compatible with that of an acute rheumatic fever. Therefore, there was a possibility that these two diseases were coexisting in this patient.

Table shows a summary of the cases of group A streptococcal endocarditis and whether or not they met the 1992 update of the Jones criteria. These reports were identified using a PubMed search with the terms "infective endocarditis" or "Streptococcus pyogenes," and we only considered reports published in English or Japanese. This search identified 21 cases among 14 reports, ranging in age from 5 months to 71 years. Streptococcal infection was confirmed using blood culture in all cases, and most cases met the major Jones criteria for endocarditis from the 1992 update. A fever and elevated acute-phase reactant levels were the main signs and symptoms in the minor criteria. Twelve of the 21 cases (as well as the present case) were also diagnosed with an acute rheumatic fever based on the 1992 update of the Jones criteria.

In conclusion, the present case highlights the difficulty in differentiating between infective endocarditis and an acute rheumatic fever as the cause of a new-onset heart murmur after a streptococcal skin and soft tissue infection. It may be impossible to differentiate between these two diseases based on the current diagnostic criteria, and to our knowledge, no reports have described the possibility of these two diseases coexisting in the same patient. Therefore, further research is needed to develop new diagnostic criteria that can differenti- ate between infective endocarditis and an acute rheumatic fever in order to facilitate determining the appropriate treatment regimen.

The authors state that they have no Conflict of Interest (COI).

\section{References}

1. Dajani AS, Ayoub E, Bierman FZ, et al. Guidelines for the diagnosis of rheumatic fever Jones criteria 1992 update. JAMA 268: 2069-2073, 1992.

2. Gewitz MH, Baltimore RS, Tani LY, et al. Revision of the Jones Criteria for the diagnosis of acute rheumatic fever in the era of Doppler echocardiography: a scientific statement from the American Heart Association. Circulation 131: 1806-1818, 2015.

3. McDonald M, Currie BJ, Carapetis JR. Acute rheumatic fever: a chink in the chain that links the heart to the throat? Lancet Infect Dis 4: 240-245, 2004.

4. Parks T, Smeesters PR, Steer AC. Streptococcal skin infection and rheumatic heart disease. Curr Opin Infect Dis 25: 145-153, 2012.

5. Li JS, Sexton DJ, Mick N, et al. Proposed modifications to the Duke criteria for the diagnosis of infective endocarditis. Clin Infect Dis 30: 633-638, 2000.

6. Selton-Suty C, Célard M, Le Moing V, et al. Preeminence of Staphylococcus aureus in infective endocarditis: a 1-year population-based survey. Clin Infect Dis 54: 1230-1239, 2012.

7. Branch J, Suganami Y, Kitagawa I, Stein GH, Tanaka E. A rare case of group A streptococcal endocarditis with absence of valvular vegetation. Intern Med 49: 1657-1661, 2010.

8. Yeşilkaya A, Azap OK, Pirat B, Gültekin B, Arslan H. A Rare 
Cause of Endocarditis: Streptococcus pyogenes. Balkan Med J 29: 331-333, 2012.

9. Lawrence JG, Carapetis JR, Griffiths K, Edwards K, Condon JR. Acute rheumatic fever and rheumatic heart disease: incidence and progression in the Northern Territory of Australia, 1997 to 2010. Circulation 128: 492-501, 2013.

10. Spinks A, Glasziou PP, Del Mar CB. Antibiotics for sore throat. Cochrane Database Syst Rev 2013.

11. Khairat $\mathrm{O}$. Myocardial abscess and acute endocarditis due to streptococcus pyogenes. Can Med Assoc J 85: 1403, 1961.

12. Cooper MJ, Silverman NH, Huey E. Group A beta-hemolytic streptococcal endocarditis precipitating rupture of sinus of Valsalva aneurysm: evaluation by two-dimensional, Doppler, and contrast echocardiography. Am Heart J 115: 1132-1134, 1988.

13. Burkert T, Watanakunakorn C. Group A streptococcus endocarditis: report of five cases and review of literature. J Infect 23: 307316, 1991.

14. Liu VC, Stevenson JG, Smith AL. Group A streptococcus mural endocarditis. Pediatr Infect Dis J 11: 1060-1062, 1992.

15. Winterbotham A, Riley S, Kavanaugh-McHugh A, Dermody TS. Endocarditis caused by group A beta-hemolytic Streptococcus in an infant: case report and review. Clin Infect Dis 29: 196-198, 1999.

16. Mohan UR, Walters S, Kroll JS. Endocarditis due to group A beta-hemolytic Streptococcus in children with potentially lethal se- quelae: 2 cases and review. Clin Infect Dis 30: 624-625, 2000.

17. Kodo K, Hida M, Omori S, et al. Vasculitis associated with septicemia: case report and review of the literature. Pediatr Nephrol 16: 1086-1092, 2001.

18. Almas A, Tariq M. Beta-hemolytic streptococcus group A endocarditis: a rare clinical presentation. J Coll Physicians Surg Pak 18: 37-39, 2008.

19. Laatiris A, Amine B, Ibn Yacoub Y, Hajjaj-Hassouni N. Septic polyarthritis caused by group A streptococcus in an immunocompetent adult: rare case. Rheumatol Int 32: 2697-2700, 2012.

20. Smith CP, Jackson C, Stewart R. Subacute bacterial endocarditis secondary to mastoiditis: a rare complication. BMJ Case Rep 2012.

21. Weidman DR, Al-Hashami H, Morris SK. Two cases and a review of Streptococcus pyogenes endocarditis in children. BMC Pediatrics 14: 227, 2014.

22. Spoladore R, Agricola E, D'Amato R, Durante A, Fragasso G, Margonato A. Isolated native tricuspid valve endocarditis due to group A beta-hemolytic Streptococcus without drug addiction. J Cardiovasc Med 16: S122-S124, 2015.

The Internal Medicine is an Open Access article distributed under the Creative Commons Attribution-NonCommercial-NoDerivatives 4.0 International License. To view the details of this license, please visit (https://creativecommons.org/licenses/ by-nc-nd/4.0/).

(C) 2017 The Japanese Society of Internal Medicine Intern Med 56: 2361-2365, 2017 